\title{
Implementación virtual de prácticas de asignaturas de control como alternativa a las prácticas de laboratorio presenciales
}

\author{
Álvaro Michelena Grandío, Francisco Zayas-Gato, Esteban Jove, José-Luis Casteleiro-Roca, \\ Héctor Quintián and José Luis Calvo-Rolle \\ University of A Coruña, CTC, CITIC, \\ Department of Industrial Engineering, Ferrol, A Coruña, Spain \\ \{alvaro.michelena, f.zayas.gato, esteban.jove, jose.luis.casteleiro, hector.quintian, jlcalvo\}@udc.es
}

\section{Resumen}

La enfermedad del coronavirus (COVID-19) ha provocado una grave crisis de salud con consecuencias socio-económicas sin precedentes. Desde la declaración de pandemia por parte de la Organización Mundial de la Salud, la mayoría de los gobiernos aplicaron políticas de control como el confinamiento, la restricción de la movilidad, el distanciamiento social, etc. En este contexto, adaptar la planificación educativa a ese escenario conlleva un gran desafío para la comunidad educativa global. Además, dado que las prácticas presenciales de laboratorio en la educación superior fueron canceladas temporalmente, la creación de escenarios virtuales realistas mediante modernas aplicaciones software representa una alternativa interesante y necesaria. Este enfoque práctico se puede lograr mediante el uso de dos herramientas de software interesantes, como Node-RED y Factory I/O. En este trabajo se propone la implementación de una planta de laboratorio virtual utilizando las herramientas mencionadas anteriormente, con características muy similares a las plantas de laboratorio reales de la Facultad de Ingeniería de la Universidad de A Coruña.

Palabras clave: Laboratorio virtual, prácticas de control y docencia en sistemas de control.

\section{Introducción}

El reciente estallido de la enfermedad del coronavirus (COVID-19) ha condicionado las metodologías de programación y enseñanza de la educación superior. Siguiendo las recomendaciones de la Organización Mundial de la Salud (OMS), los gobiernos de la mayoría de los países decretaron restricciones de movilidad y distanciamiento social para prevenir la transmisión del virus. Además, se cerraron muchas instalaciones universitarias en un intento por controlar la propagación del virus. En consecuencia, las clases presenciales fueron suspendidas temporalmente y la enseñanza online se convirtió en obligatoria, lo que implicó un cambio dr ástico en un periodo de tiempo muy corto. Esta situación ha impactado dramáticamente a la comunidad educativa en todo el mundo [35, 15, 20, 19].

Uno de los campos más afectados en la educación en ingeniería has sido la práctica de laboratorio así como su planificación. Este es un punto clave, ya que las clases de teoría se complementan con prácticas de laboratorio en la mayoría de las asignaturas. Esta metodología fomenta el aprendizaje colaborativo promoviendo el aprendizaje basado en proyectos.

Generalmente, los laboratorios tienen equipos específicos dedicados para clases prácticas. Sin embargo, en muchos casos, debido a las limitaciones del material disponible, es necesario organizar grupos de trabajo para realizar las tareas. En este contexto, debido a la naturaleza infecciosa del coronavirus, los grupos de trabajo ahora se han reducido o incluso has sido prohibidos para prevenir la transmisión del virus. De ahí que muchos profesores se esforzaran por rediseñar sus métodos de enseñanza, apoyándose en recursos tecnológicos para ofrecer una alternativa a los laboratorios reales [38, 37, 25, 3, 46].

Las lecciones de laboratorio fomentan la interacción de los estudiantes y el manejo de equipos físicos. Si bien las lecciones teóricas online son una alternativa a las presenciales, acceder a los laboratorios de forma remota no es una práctica común y no es posible en muchos casos. Es por ello que, una alternativa interesante a los laboratorios reales son los laboratorios virtuales. Hoy en día, muchos de los sistemas basados en técnicas de inteligencia artificial como los que se explican en $[31,1,9,17,39,7,28,26,33,13,45,29,36,40$, $44,34,27,4,47,4,16,14]$ se pueden verificar, en las primeras fases de diseño, mediante este tipo de laboratorios.

Hoy en día, gracias al progreso constante en computación y digitalización, muchas herramientas software pueden ser usadas para implementar escenarios virtuales. $[42,11,12,22,24,30]$. En este trabajo se propone la integración de las herramientas de software Node-RED y Factory I/O para crear un escenario virtual lo más parecido posible a las plantas de control de nivel reales del 
Laboratorio de Optimización y Control de la Facultad de Ingeniería de la Universidad de A Coruña (UDC).

\section{Contexto}

El Laboratorio de Optimización y Control es uno de los laboratorios de la Facultad de Ingeniería de la Universidad de A Coruña (UDC). Este laboratorio cuenta con algunos equipos que emulan muchos procesos industriales reales, como lazos de control de temperatura y nivel de agua. En él, los estudiantes realizan prácticas de varias asignaturas de diferentes cursos con diversos niveles de dificultad, interactúan con el equipo, experimentan con sensores y actuadores, y prueban sus proyectos finales de grado y máster.

Uno de los equipos de laboratorio es el sistema de control de temperatura. Este sistema esta formado por un cubo de metracrilato, en cuyo interior dos bombillas incandescentes actúan como elemento calefactor. Además, un ventilador de CC (corriente continua) actúa como un elemento enfriador (ver Figura 1). Para medir la temperatura en el interior del cubo se usa un sensor de temperatura LM35. La adquisición de datos de esta planta se puede realizar con un dispositivo DAQ (sistema de adquisición de datos) multifunción USB-6008 de National Instruments (ver Figura 2).

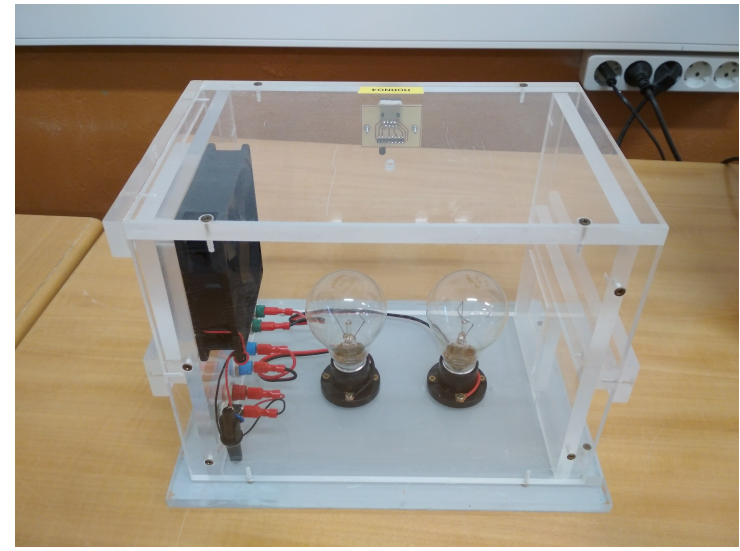

Figura 1: Planta de control de temperatura

Los estudiantes pueden organizarse por parejas o individualmente para trabajar con los sistemas de control de temperatura, porque hay suficientes unidades para este propósito.

Otro de los equipos disponibles en el laboratorio son los diversos sistema de control de nivel en depósitos disponibles ??. A diferencia de los sistemas de control de temperatura, hay menos plantas de control de nivel disponibles. Es por ello que los grupos de trabajo de estudiantes deben ser

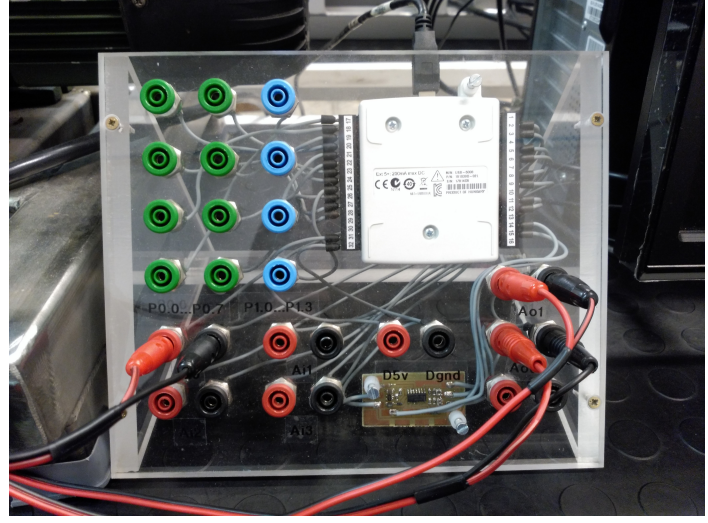

Figura 2: Tarjeta de adquisición de datos

más grandes. Además de lo anterior, es necesario tener en cuenta que se debe respetar el distanciamiento social debido a la pandemia de COVID-19 $[41,32,6,23,10,8]$.

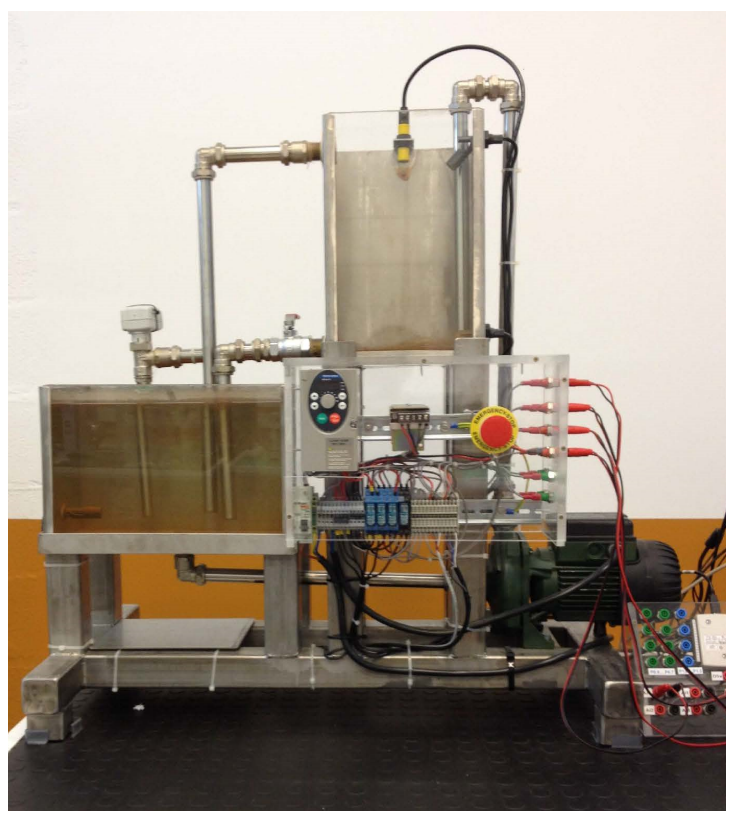

Figura 3: Planta de control de nivel

\section{Caso de estudio}

Esta sección describe la planta de control de nivel así como el y el circuito de control que se implementará virtualmente.

\subsection{Planta de control de nivel}

Como se mencionó anteriormente, uno de los sistemas disponibles en el Laboratorio de Optimización y Control es el circuito de control del nivel de depósitos, que se utiliza para fijar el nivel del líquido en un depósito o tanque.

El sistema (ver Figura 3) tiene dos depósitos a 


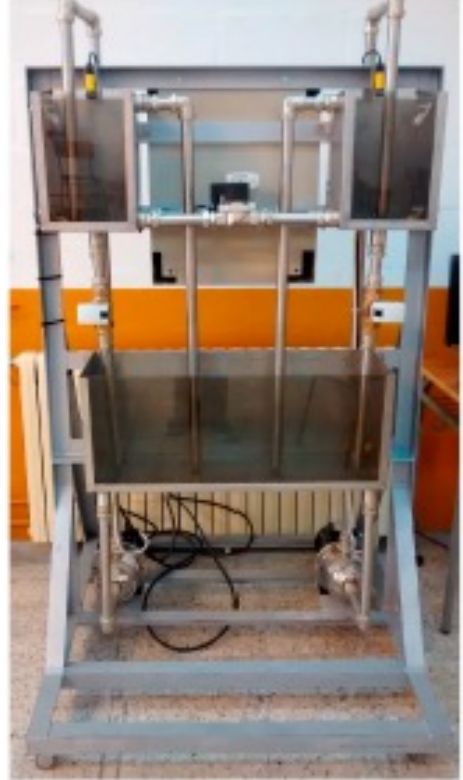

Figura 4: Planta de control de nivel doble

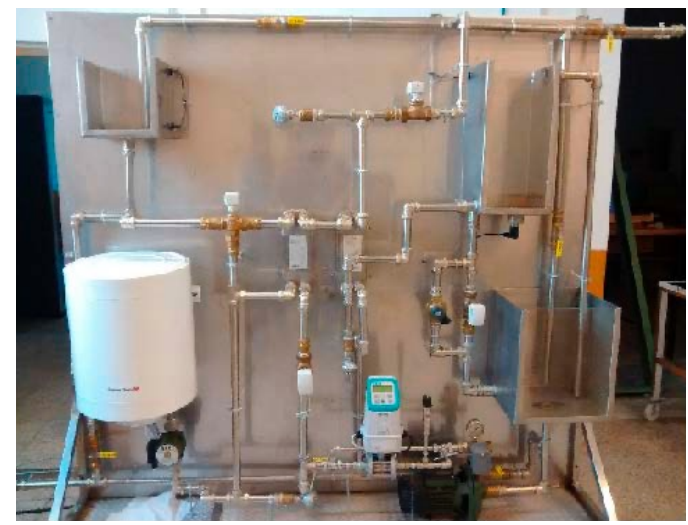

Figura 5: Planta de control de nivel con intercambiadores

diferentes niveles. El depósito inferior se utiliza para almacenar el agua que se usa para llenar y descargar el depósito superior, que se utiliza como objetivo de control de nivel de líquido. Se utiliza una bomba centrífuga alimentada desde el depósito inferior para bombear agua al depósito superior. Para vaciarlo, se puede utilizar una válvula proporcional tanto manual como servopilotada. Un variador de frecuencia (VFD), conectado al motor trifásico de la bomba, controla la velocidad de llenado del depósito superior. Además, el nivel del agua en este depósito se mide con un sensor ultrasónico. Como DAQ se utiliza un Arduino Uno para adquirir las señales del proceso.

\subsection{Sistema de control}

El sistema de control es un controlador digital implementado por software, desarrollado usando un

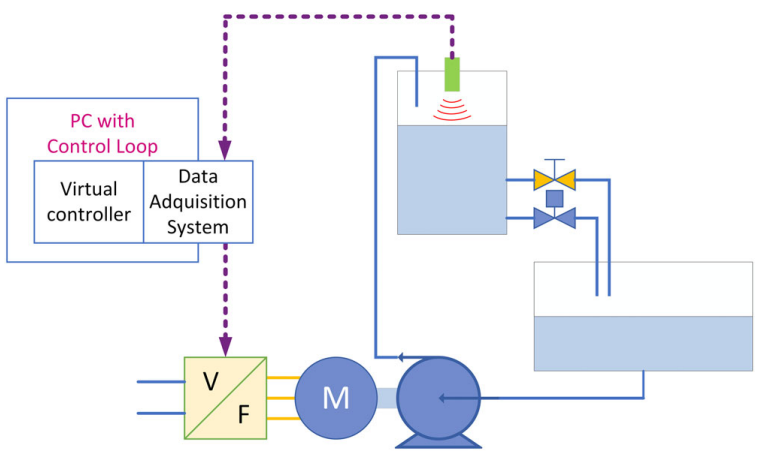

Figura 6: Esquema simplificado de la planta de control de nivel

ordenador y un DAQ (ver Figuras 6 y 7). Se basa en un controlador PID, siendo la consigna el nivel de agua deseado y la variable de proceso el nivel de agua actual medido en el depósito superior. El nivel del agua se mide con el sensor ultrasónico que envía un voltaje al DAQ. Gracias a eso, la ordenador recibe y procesa la señal recibiendo así el nivel de agua en un rango 0\% (vacío) a $100 \%$ lleno.

La señal de error (consigna menos nivel actual) es la entrada al controlador digital, el cual calcula la señal de control y la envía a través de una de las salidas analógicas del DAQ al VFD de la planta, cerrando el circuito de control de nivel.

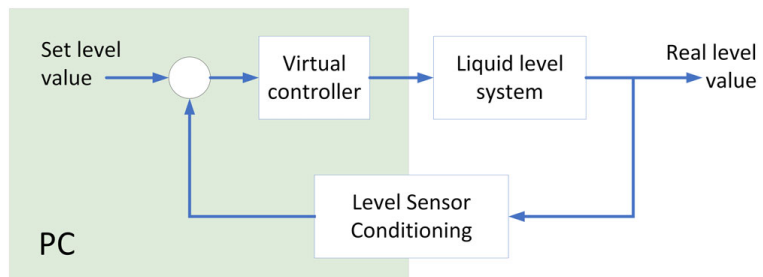

Figura 7: Arquitectura del sistema de control de nivel

\section{Integración de herramientas}

Dado que la planta de control de nivel del Laboratorio de Optimización y Control no está disponible para operar debido a la pandemia COVID-19, se propone el uso de un modelo virtual del sistema real $[42,21,2,43,18,5]$.

Esta sección presenta las herramientas de software que se utilizan para crear una planta de control de nivel virtual, así como el sistema de control virtual implementado. 


\subsection{Planta de nivel virtual con Factory I/O}

Factory I/O es un software de diseño 3D para simulación de fábrica. Es una herramienta con gran potencia para complementar el aprendizaje de la ingeniería de automatización y control. Como software de fácil uso, Factory I/O permite a los estudiantes crear escenarios virtuales rápidamente y comprender el funcionamiento de la fábrica desde un punto de vista más realista (Figura 8).

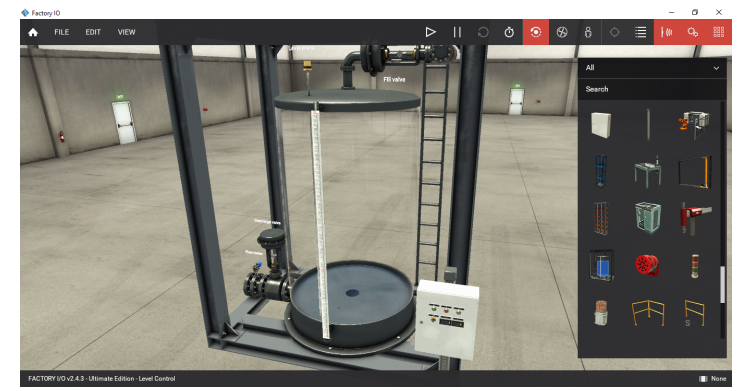

Figura 8: Planta de nivel con Factory I/O

Factory I/O dispone de muchos de los elementos más comunes de la industria modelados y virtualizados. Algunos de ellos son: cintas transportadoras, cuadros eléctricos, elevadores, paletizadores, cisternas, sensores, etc. Todos estos elementos se pueden ensamblar para configurar un proceso industrial específico y así construir una fábrica virtual.

Uno de los escenarios más interesantes es el uso de Factory I/O como plataforma de validación para sistemas de control basados en PLC. Permite probar cómo se comportaría un proceso industrial antes de ser implementado en un entorno real. Además, cuenta con una amplia gama de protocolos de comunicación industrial, permitiendo conexiones con PLC, microcontroladores, Ordenadores de Placa Única (SBC) o incluso con otro tipo de aplicaciones de escritorio.

La planta de control de nivel disponible en Factory I/O y la planta de control de nivel real del Laboratorio de Optimización y Control tienen muchas características en común. Además de tener un tanque para fijar el nivel del agua, la planta virtual de control de nivel cuenta con sensores y actuadores con entradas y salidas analógicas industriales. Sin embargo, estas señales de entrada y salida no están disponibles por hardware. Por lo tanto, una interfaz entre la planta virtual y el controlador real no es posible utilizando una tarjeta de adquisición de datos de hardware.

Una alternativa a la tarjeta de adquisición hardware es una interfaz de comunicación industrial. Factory I/O tiene varios controladores de comu- nicación estándar para leer y escribir las señales de entrada y salida generadas por las sistemas virtuales. Algunos de estos controladores de comunicación son Modbus TCP Client, Modbus TCP Server, OPC Client DA/UA, Siemens S71200/1500, etc. Por lo tanto, cualquier aplicación software compatible con dichos controladores de comunicación se puede utilizar como alternativa a un sistema basado en DAQ.

\subsection{Interfaz y control con Node-RED}

Node-RED es un software de programación gráfica para conectar dispositivos de hardware y también establecer comunicaciones entre aplicaciones. Desarrollado originalmente por el equipo de servicios de tecnología emergente de IBM, Node-RED es ahora parte de la Fundación OpenJS.

La programación gráfica, o, en este caso, la programación "basada en flujo", es una forma de desarrollar aplicaciones interconectando bloques o "nodos" (ver Figura 9). Node-RED no solo ofrece una paleta de nodos predefinidos, también tiene la posibilidad de personalizar los nodos para realizar funciones específicas. Cada nodo dentro de la aplicación recibe datos a través de sus entradas, ejecuta acciones basadas en esos datos y envía datos a través de sus salidas. La forma y el orden de las interconexiones de nodos definen el comportamiento de la aplicación.

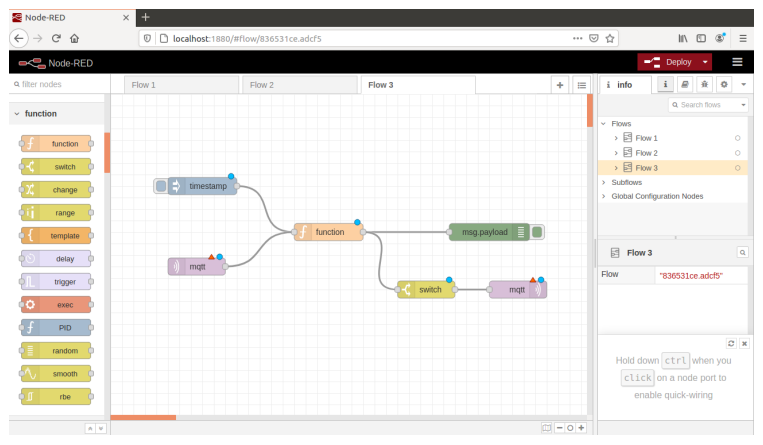

Figura 9: Entorno de programación de Node-RED

El entorno de desarrollo integrado (IDE) está basado en un servidor web, por lo que no es necesario instalar ninguna aplicación de escritorio dedicada. Solo se requiere un navegador web para acceder al entorno de programación. Por lo tanto, para desarrollar aplicaciones para un dispositivo conectado a una red, se puede utilizar un teléfono inteligente, una tableta, un ordenador portátil, etc.

Node-RED facilita el desarrollo de aplicaciones y ayuda a comprender el orden de ejecución del programa. Puede considerarse como una herramienta de apoyo, por lo tanto, los estudiantes no deben 
de preocuparse por dominar un nuevo lenguaje de programación.

El aspecto clave de utilizar Node-RED para esta propuesta es que sirve como el primer paso del enfoque de integración. Además, al contar con los controladores de comunicación necesarios, es posible la implementación de un controlador PID virtual.

Sin embargo uno de los aspectos más interesantes es la facilidad con la que se puede hacer la integración con otros programas de control de procesos de los que ya se disponga a través de una comunicación Modbus TCP en tiempo real.

Ello permite usar programas ya diseñados y que funcionen en procesos reales sobre los mismos procesos pero virtualizados, para comprobar el grado de similitud entre ambos.

\section{Solución implementada}

Esta sección describe los métodos para lograr la integración de la aplicación y la solución virtual desarrollada como una alternativa al actual sistema de plantas de control de nivel real.

\subsection{Comunicación de aplicaciones a través de Modbus TCP}

Modbus es uno de los protocolos de comunicación más populares de la industria. Después de más de cuarenta años desde su publicación, todavía se usa ampliamente para conectar millones de dispositivos en todo el mundo. Es un estándar industrial de facto creado por Modicon y posteriormente adoptado por muchos otros fabricantes ya que no tiene derechos de autor.

Modbus es un protocolo robusto y fácil de implementar, ofreciendo, además, una arquitectura cliente/servidor que permite la conexión de múltiples dispositivos a través de un bus de campo. También admite múltiples versiones como Modbus RTU, Modbus ASCII, Modbus TCP, etc. Independientemente de la versión utilizada, el objetivo principal es el intercambio de datos entre dispositivos o aplicaciones.

Factory I/O y Node-RED disponen de controladores para comunicarse a través de Modbus TCP. Estas aplicaciones pueden utilizar este protocolo de comunicación para realizar el intercambio de datos. Por lo tanto, las señales de control y varible de proceso del controlador y la planta virtual se pueden enviar a través de este enlace. En este caso, el lado del cliente Modbus lo realiza la aplicación Node-RED y el lado del servidor por la planta virtual de nivel como se puede ver en las
Figuras 10 y 11.

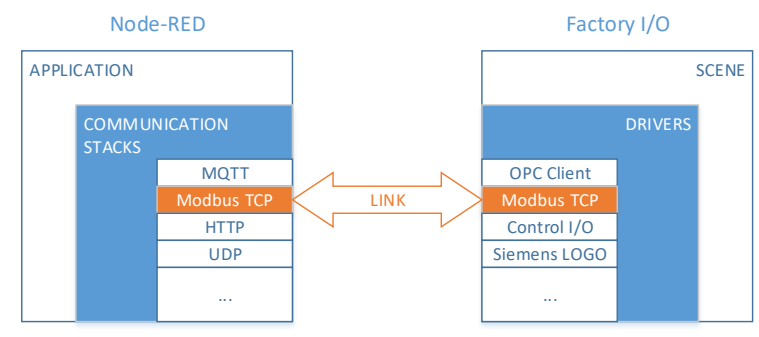

Figura 10: Enlace de comunicación entre NodeRED y Factory I/O

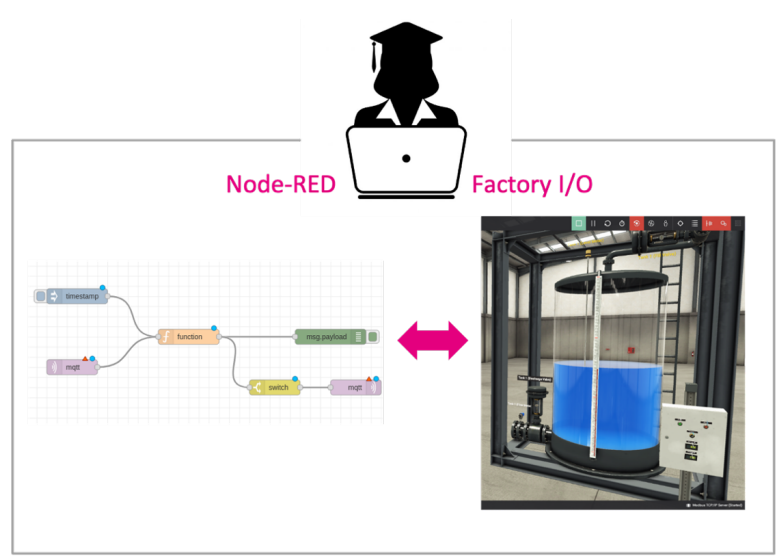

Figura 11: Integración de Node-RED en un único equipo

\subsection{Contro PID con Node-RED}

Como se mencionó anteriormente, el sistema de control de la planta de nivel real se basa en un controlador PID desarrollado con Matlab/Simulink, el cual si bien dispone de herramientas para diseñar y ajustar controladores PID, este tiene algunas limitaciones en términos de interfaces de comunicación industrial.

Por otro lado, Node-RED dispone de algunos nodos predefinidos que implementan algoritmos de control PID así como nodos de interfaz de comunicación. Esto permite la integración de la comunicación y el control en el mismo paquete de software. Similar a un bloque PID Matlab/Simulink, un nodo de control PID recibe la consigna y la variable de proceso a través de sus entradas y envía la señal de control a través de su salida. Luego, los usuarios pueden concentrarse en aprender y ajustar el controlador, en lugar de codificar el algoritmo PID por sí mismos.

La variable de proceso de la planta virtual y la señal de control PID de Node-RED se pueden intercambiar utilizando la interfaz Modbus TCP. Esto hace posible cerrar el circuito de control y 
ejecutar el proceso en un solo ordenador (Figura 12). El sistema implementado consiste en un depósito de agua que mediante un regulador PID permite controlar un nivel determinado fijado por el usuario.

La evolución de la respuesta del sistema es mostrada mediante la interfaz gráfica incluida también en la figura 12. Dicha interfaz es totalmente configurable, permitiendo mostrar otras variables en tiempo real.

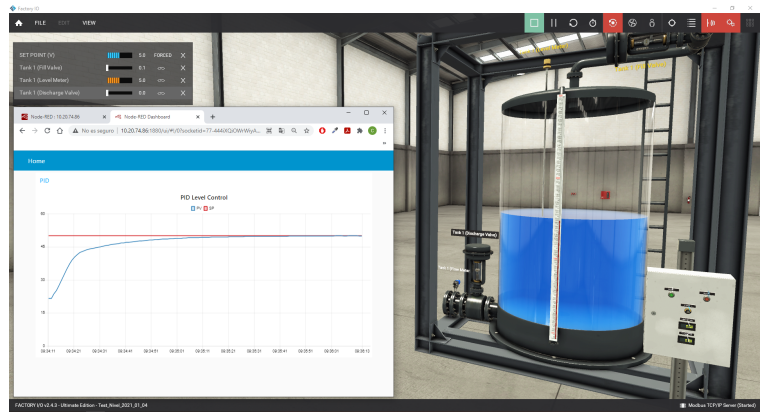

Figura 12: Integración de Node-RED y Factory $\mathrm{I} / \mathrm{O}$

\subsection{Visualización, análisis y resultados}

El funcionamiento de la planta virtual creada con Factory I/O se puede visualizar en tiempo real una vez cerrado el lazo de control. Antes de esto, es necesario ejecutar ambas aplicaciones y configurar los parámetros de la interfaz de comunicación. Cada aplicación debe fijar su propia dirección IP y puerto, así como el mapa de memoria requerido para el intercambio de datos. Además, es importante verificar la escala de todas las variables del sistema para garantizar un funcionamiento correcto.

La visualización de la respuesta de la planta virtual es un aspecto clave. De hecho, un tema de la ingeniería de control es el ajuste empírico de los controladores PID en función de los parámetros de respuesta en el dominio del tiempo. Las técnicas de ajuste de PID de bucle abierto y de bucle cerrado, así como los métodos de oscilación sostenida o "relay-feedback", son ampliamente aceptadas y siguen utilizándose debido a su simplicidad. El ajuste de los controladores PID con estas técnicas se basa en una representación precisa de la respuesta de la planta. Por lo tanto, se requiere una herramienta de adquisición y visualización de datos.

Node-RED tiene un subconjunto de nodos nodered-dashboard para trazar datos en tiempo real. Además de gráficos circulares, gráficos de líneas e indicadores, este subconjunto también tiene elementos de interfaz de usuario como botones, con- troles deslizantes, entradas de texto, etc. Combinando estos nodos con las funciones de control y comunicación, se puede implementar una interfaz gráfica de usuario completa (GUI) junto con un conjunto de análisis y control, como la mostrada en la figura 12, en la que se grafica tanto la acción de control, el error y el setpoint, despuás de diseñar y aplicar un regulador PID ajustado mediante el método de "relay-feedback" en cadena cerrada.

\section{Conclusiones y trabajos futuros}

Este trabajo presenta una solución para impartir prácticas de laboratorio online, como alternativa a las clases presenciales con una planta de laboratorio real. Además, aprovechando la tecnología de la información (TI) y las herramientas de software modernas, los estudiantes pueden simplemente diseñar su propia planta de laboratorio virtual y realista, trabajar individualmente y compartir los resultados con sus compañeros y profesores.

Todo ello permite que los estudiantes pongan en práctica sus conocimientos teóricos aumentando de forma significativa el numero de horas que pueden dedicar a la parte práctica de las asignaturas en un entorno virtual muy similar a uno industrial, facilitando de este modo el enfrentarse posteriormente a las plantas industriales reales.

Por otro lado, también sirve como solución a la problemática derivada de la situación de restricción de capacidades como consecuencia del COVID-19, lo que limita de forma significativa el número de puestos disponibles para realización de prácticas, así como posibilita una ampliación de los horarios, de forma no presencial, en el que los alumnos pueden realizar tanto sus prácticas como sus trabajos sobre procesos industriales simulados pero muy realistas.

El sistema virtual, no solo permite una interacción en tiempo real de forma visual, sino que también dispone de sonido en tiempo real, de forma que la experiencia es todavía más inmersiva.

Dada la similitud entre el sistema virtual con el caso real mostrado en el apartado de solución implementada, se puede concluir que esta propuesta se presenta como un método interesante para evitar un cambio mayor en la programación de las prácticas de laboratorio como consecuencia de la pandemia COVID-19.

Después de validar la solución propuesta, podemos concluir que se han obtenido muy buenos resulta- 
dos. Además, se ha logrado una gran aceptación por parte de estudiantes y profesores.

Desde el punto de vista de los estudiantes cabe destacar una mayor implicación por su parte al disponer de una mayor flexibilidad de horarios

Desde el punto de vista de los docentes, lo más destacable es la flexibilidad del escenarios que se pueden plantear, pudiendo configurar desde sistema industriales sencillos, hasta otros más complejos con múltiples variables de control y medida.

Como trabajos futuros, la virtualización de otras plantas de laboratorio y sistemas como lazos de control de temperatura, podrían ayudar a mitigar el impacto de la pandemia en la modalidad presencial. Además, los estudiantes también se benefician al aprender otras herramientas de software de gran utilidad.

\section{English summary}

\section{VIRTUAL IMPLEMENTATION OF PRACTICES OF CONTROL SUB- JECTS AS AN ALTERNATIVE TO FACE-TO-FACE LABORATORY PRACTICES}

\author{
Abstract \\ The coronavirus disease (COVID-19) has \\ caused a serious health crisis with un- \\ precedented socio-economic consequences. \\ Since the declaration of a pandemic by the \\ World Health Organization, most govern- \\ ments have applied control policies such as \\ confinement, restriction of mobility, social \\ distancing, etc. In this context, adapt- \\ ing educational planning to this scenario \\ poses a great challenge for the global ed- \\ ucational community. In addition, given \\ that the face-to-face laboratory practices \\ in higher education were temporarily can- \\ celed, the creation of realistic virtual sce- \\ narios using modern software applications \\ represents an interesting and necessary al- \\ ternative. This practical approach can be \\ achieved by using two interesting software \\ tools, such as Node-RED and Factory I/ \\ O. In this work, the implementation of a \\ virtual laboratory plant is proposed using
}

the tools mentioned above, with characteristics very similar to the real laboratory plants of the Faculty of Engineering of the University of A Coruña.

Keywords: Virtual laboratory, control practices and teaching in control systems.

\section{Referencias}

[1] Aláiz-Moretón, H., Castejón-Limas, M., Casteleiro-Roca, J.L., Jove, E., Fernández Robles, L., Calvo-Rolle, J.L.: A fault detection system for a geothermal heat exchanger sensor based on intelligent techniques. Sensors 19(12), 2740 (2019)

[2] Alaiz-Moretón, H., Jove, E., Casteleiro-Roca, J.L., Quintián, H., López García, H., BenítezAndrades, J.A., Novais, P., Calvo-Rolle, J.L.: Bioinspired hybrid model to predict the hydrogen inlet fuel cell flow change of an energy storage system. Processes 7(11), 825 (2019)

[3] Basurto, N., Arroyo, Á., Vega, R., Quintián, H., Calvo-Rolle, J.L., Herrero, Á.: A hybrid intelligent system to forecast solar energy production. Computers \& Electrical Engineering 78, 373-387 (2019)

[4] Calvo-Rolle, J.L.: Data collection description for evaluation and analysis of engineering students academic performance. In: The 11th International Conference on EUropean Transnational Educational (ICEUTE 2020). vol. 1266, p. 317. Springer Nature (2020)

[5] Calvo-Rolle, J.L., Casteleiro-Roca, J.L., Quintián, H., del Carmen Meizoso-Lopez, M.: A hybrid intelligent system for pid controller using in a steel rolling process. Expert Systems with Applications 40(13), 5188-5196 (2013)

[6] Casteleiro-Roca, J.L., Barragán, A.J., Segura, F., Calvo-Rolle, J.L., Andújar, J.M.: Fuel cell output current prediction with a hybrid intelligent system. Complexity 2019 (2019)

[7] Casteleiro-Roca, J.L., García-Ordás, M.T., Jove, E., Zayas-Gato, F., Quintián, H., AlaizMoretón, H., Calvo-Rolle, J.L.: A solar thermal system temperature prediction of a smart building for data recovery and security purposes. In: International Conference on Intelligent Data Engineering and Automated Learning. pp. 468-476. Springer (2020) 
[8] Casteleiro-Roca, J.L., Gómez-González, J.F., Calvo-Rolle, J.L., Jove, E., Quintián, H., Martín, J.F.A., Perez, S.G., Diaz, B.G., Calero-Garcia, F., Méndez-Perez, J.A.: Prediction of the energy demand of a hotel using an artificial intelligence-based model. In: International conference on hybrid artificial intelligence systems. pp. 586-596. Springer (2018)

[9] Casteleiro-Roca, J.L., Javier Barragan, A., Segura, F., Luis Calvo-Rolle, J., Manuel Andujar, J.: Intelligent hybrid system for the prediction of the voltage-current characteristic curve of a hydrogen-based fuel cell. Revista Iberoamericana de Automática e Informática industrial 16(4), 492-501 (2019)

[10] Crespo-Turrado, C., Casteleiro-Roca, J.L., Sánchez-Lasheras, F., López-Vázquez, J.A., De Cos Juez, F.J., Pérez Castelo, F.J., CalvoRolle, J.L., Corchado, E.: Comparative study of imputation algorithms applied to the prediction of student performance. Logic Journal of the IGPL 28(1), 58-70 (2020)

[11] Daniel, J.: Education and the covid-19 pandemic. Prospects 49(1), 91-96 (2020)

[12] Fernández-Serantes, L.A., Vázquez, R.E., Casteleiro-Roca, J.L., Calvo-Rolle, J.L., Corchado, E.: Hybrid intelligent model to predict the soc of a lfp power cell type. In: International conference on hybrid artificial intelligence systems. pp. 561-572. Springer (2014)

[13] Gonzalez-Cava, J.M., Arnay, R., Pérez, J.A.M., León, A., Martín, M., Jove-Perez, E., Calvo-Rolle, J.L., Casteleiro-Roca, J.L., de Cos Juez, F.J.: A machine learning based system for analgesic drug delivery. In: International Joint Conference SOCO'17CISIS'17-ICEUTE'17 León, Spain, September 6-8, 2017, Proceeding. pp. 461-470. Springer (2017)

[14] Gonzalez-Cava, J.M., Reboso, J.A., Casteleiro-Roca, J.L., Calvo-Rolle, J.L., Méndez Pérez, J.A.: A novel fuzzy algorithm to introduce new variables in the drug supply decision-making process in medicine. Complexity 2018 (2018)

[15] Jena, P.K.: Impact of covid-19 on higher education in india. International Journal of Advanced Education and Research (IJAER) 5 (2020)

[16] Jove, E., Casteleiro-Roca, J., Quintián, H., Méndez-Pérez, J., Calvo-Rolle, J.: Anomaly detection based on intelligent techniques over a bicomponent production plant used on wind generator blades manufacturing. Revista Iberoamericana de Automática e Informática industrial 17(1), 84-93 (2020)

[17] Jove, E., Aláiz-Moretón, H., Casteleiro-Roca, J.L., Corchado, E., Calvo-Rolle, J.L.: Modeling of bicomponent mixing system used in the manufacture of wind generator blades. In: International Conference on Intelligent Data Engineering and Automated Learning. pp. 275-285. Springer (2014)

[18] Jove, E., Alaiz-Moretón, H., GarcíaRodríguez, I., Benavides-Cuellar, C., Casteleiro-Roca, J.L., Calvo-Rolle, J.L.: Pid-its: an intelligent tutoring system for pid tuning learning process. In: International Joint Conference SOCO'17-CISIS'17ICEUTE'17 León, Spain, September 6-8, 2017, Proceeding. pp. 726-735. Springer (2017)

[19] Jove, E., Blanco-Rodríguez, P., CasteleiroRoca, J.L., Moreno-Arboleda, J., LópezVázquez, J.A., de Cos Juez, F.J., CalvoRolle, J.L.: Attempts prediction by missing data imputation in engineering degree. In: International Joint Conference SOCO'17CISIS'17-ICEUTE'17 León, Spain, September 6-8, 2017, Proceeding. pp. 167-176. Springer (2017)

[20] Jove, E., Blanco-Rodríguez, P., CasteleiroRoca, J.L., Quintián, H., Moreno Arboleda, F.J., LóPez-Vázquez, J.A., RodríguezGómez, B.A., Meizoso-López, M.D.C., Piñón-Pazos, A., De Cos Juez, F.J., et al.: Missing data imputation over academic records of electrical engineering students. Logic Journal of the IGPL 28(4), 487-501 (2020)

[21] Jove, E., Casteleiro-Roca, J.L., Quintián, H., Méndez-Pérez, J.A., Calvo-Rolle, J.L.: A new approach for system malfunctioning over an industrial system control loop based on unsupervised techniques. In: The 13th International Conference on Soft Computing Models in Industrial and Environmental Applications. pp. 415-425. Springer (2018)

[22] Jove, E., Casteleiro-Roca, J.L., Quintián, H., Méndez-Pérez, J.A., Calvo-Rolle, J.L.: Outlier generation and anomaly detection based on intelligent one-class techniques over a bicomponent mixing system. In: International Workshop on Soft Computing Models in Industrial and Environmental Applications. pp. 399-410. Springer (2019) 
[23] Jove, E., Casteleiro-Roca, J.L., Quintián, H., Méndez-Pérez, J.A., Calvo-Rolle, J.L.: Virtual sensor for fault detection, isolation and data recovery for bicomponent mixing machine monitoring. Informatica 30(4), 671-687 (2019)

[24] Jove, E., Casteleiro-Roca, J.L., Quintián, H., Méndez-Pérez, J.A., Calvo-Rolle, J.L.: A new method for anomaly detection based on non-convex boundaries with random twodimensional projections. Information Fusion 65, 50-57 (2021)

[25] Jove, E., Casteleiro-Roca, J.L., Quintián, H., Simić, D., Méndez-Pérez, J.A., Luis CalvoRolle, J.: Anomaly detection based on oneclass intelligent techniques over a control level plant. Logic Journal of the IGPL 28(4), 502518 (2020)

[26] Jove, E., Casteleiro-Roca, J.L., Quintián, H., Zayas-Gato, F., Casado-Vara, R., Baruque, B., Méndez-Pérez, J.A., Calvo-Rolle, J.L.: A hybrid one-class topology for non-convex sets. In: International Conference on Hybrid Artificial Intelligence Systems. pp. 341-349. Springer (2020)

[27] Jove, E., Casteleiro-Roca, J.L., Quintián, H., Zayas-Gato, F., Novais, P., Méndez-Pérez, J.A., Calvo-Rolle, J.L.: A comparative study to detect flowmeter deviations using one-class classifiers. In: Computational Intelligence in Security for Information Systems Conference. pp. 66-75. Springer (2019)

[28] Jove, E., Casteleiro-Roca, J.L., Quintián, H., Zayas-Gato, F., Vercelli, G., Calvo-Rolle, J.L.: A one-class classifier based on a hybrid topology to detect faults in power cells. Logic Journal of the IGPL (2021)

[29] Jove, E., Gonzalez-Cava, J.M., CasteleiroRoca, J.L., Quintián, H., Méndez-Pérez, J.A., Calvo-Rolle, J.L.: Anomaly detection on patients undergoing general anesthesia. In: International Joint Conference: 12th International Conference on Computational Intelligence in Security for Information Systems (CISIS 2019) and 10th International Conference on EUropean Transnational Education (ICEUTE 2019). pp. 141-152. Springer (2019)

[30] Jove, E., Gonzalez-Cava, J.M., CasteleiroRoca, J.L., Quintián, H., Méndez Pérez, J.A., Vega Vega, R., Zayas-Gato, F., de Cos Juez, F.J., León, A., MartÍn, M., et al.: Hybrid intelligent model to predict the remifentanil infusion rate in patients under general anesthesia. Logic Journal of the IGPL 29(2), 193206 (2021)

[31] Jove, E., Vázquez, J.A.L., Fernández-Ibáñez, I., Casteleiro-Roca, J.L., Rolle, J.L.C.: Hybrid intelligent system to predict the individual academic performance of engineering students. The International journal of engineering education 34(3), 895-904 (2018)

[32] Laal, M., Laal, M.: Collaborative learning: what is it? Procedia-Social and Behavioral Sciences 31, 491-495 (2012)

[33] Leira, A., Jove, E., Gonzalez-Cava, J.M., Casteleiro-Roca, J.L., Quintián, H., ZayasGato, F., Álvarez, S.T., Simić, S., MéndezPérez, J.A., Luis Calvo-Rolle, J.: Oneclass-based intelligent classifier for detecting anomalous situations during the anesthetic process. Logic Journal of the IGPL (2020)

[34] Luis Casteleiro-Roca, J., Quintián, H., Luis Calvo-Rolle, J., Méndez-Pérez, J.A., Javier Perez-Castelo, F., Corchado, E.: Lithium iron phosphate power cell fault detection system based on hybrid intelligent system. Logic Journal of the IGPL 28(1), 71-82 (2020)

[35] Marinoni, G., Van't Land, H., Jensen, T.: The impact of covid-19 on higher education around the world. IAU Global Survey Report (2020)

[36] Martinez-Álvarez, F., Troncoso, A., Quintián, H., Corchado, E.: A data structure to speed-up machine learning algorithms on massive datasets francisco. In: International Conference on Hybrid Artificial Intelligence Systems. vol. 9648, pp. 365-376. Springer, Cham, (2016)

[37] Nicola, M., Alsafi, Z., Sohrabi, C., Kerwan, A., Al-Jabir, A., Iosifidis, C., Agha, M., Agha, R.: The socio-economic implications of the coronavirus and covid-19 pandemic: a review. International journal of surgery (2020)

[38] Pérez-López, E., Atochero, A.V., Rivero, S.C.: Educación a distancia en tiempos de covid-19: Análisis desde la perspectiva de los estudiantes universitarios. Revista Iberoamericana de Educación a Distancia 24(1), 331-350 (2021)

[39] Quintián, H., Casteleiro-Roca, J.L., PerezCastelo, F.J., Calvo-Rolle, J.L., Corchado, E.: Hybrid intelligent model for fault detection of a lithium iron phosphate power 
cell used in electric vehicles. In: International conference on hybrid artificial intelligence systems. pp. 751-762. Springer (2016)

[40] Quintián, H., Rolle, J.L.C., Romero, Ó.F.: Aplicación de un robot comercial de bajo coste en tareas de seguimiento de objetos. DYNA: revista de la Facultad de Minas. Universidad Nacional de Colombia. Sede Medellín 79(175), 24-33 (2012)

[41] Ray, S., Srivastava, S.: Virtualization of science education: a lesson from the covid-19 pandemic. Journal of proteins and proteomics 11, 77-80 (2020)

[42] Vasiliadou, R.: Virtual laboratories during coronavirus (covid-19) pandemic. Biochemistry and Molecular Biology Education 48(5), 482-483 (2020)

[43] Vega Vega, R., Quintián, H., Calvo-Rolle, J.L., Herrero, Á., Corchado, E.: Gaining deep knowledge of android malware families through dimensionality reduction techniques. Logic Journal of the IGPL 27(2), 160-176 (2019)

[44] Vega Vega, R., Quintián, H., Cambra, C., Basurto, N., Herrero, Á., Calvo-Rolle, J.L.: Delving into android malware families with a novel neural projection method. Complexity 2019 (2019)

[45] Vega Vega, R.A., Chamoso-Santos, P., González Briones, A., Casteleiro-Roca, J.L., Jove, E., Meizoso-López, M.d.C., RodríguezGómez, B.A., Quintián, H., Herrero, Á., Matsui, K., et al.: Intrusion detection with unsupervised techniques for network management protocols over smart grids. Applied Sciences 10(7), 2276 (2020)

[46] Zayas-Gato, F., Quintián, H., Jove, E., Casteleiro-Roca, J.L., Calvo-Rolle, J.L.: Diseño de controladores PID. Universidade da Coruña, Servizo de Publicacións (2020)

[47] Zayas-Gato, F., Simic, D., Calvo-Rolle, J.L.: Hybrid approximate convex hull one-class classifier for an industrial plant. In: 13th International Conference on Computational Intelligence in Security for Information Systems (CISIS 2020). vol. 1267, p. 282. Springer Nature (2020)

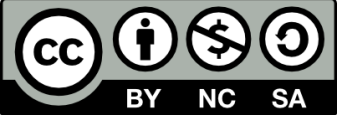

C 2021 by the authors. Submitted for possible open access publication under the terms and conditions of the Creative Commons Attribution CC BY-NC-SA 4.0 license (https://creativecommons.org/licenses/by ncsa 